\title{
Development of Galerkin Method for Solving the Generalized Burger's-Huxley Equation
}

\author{
M. El-Kady, ${ }^{1}$ S. M. El-Sayed, ${ }^{2}$ and H. E. Fathy ${ }^{3}$ \\ ${ }^{1}$ Department of Mathematics, Faculty of Science, Helwan University, Cairo, Egypt \\ ${ }^{2}$ Department of Scientific Computing, Faculty of Computers and Informatics, Benha University, Benha 13518, Egypt \\ ${ }^{3}$ Department of Mathematics, Faculty of Science, Benha University, Benha, Egypt
}

Correspondence should be addressed to M. El-Kady; mam_el_kady@yahoo.com

Received 13 November 2012; Accepted 6 January 2013

Academic Editor: Hung Nguyen-Xuan

Copyright (C) 2013 M. El-Kady et al. This is an open access article distributed under the Creative Commons Attribution License, which permits unrestricted use, distribution, and reproduction in any medium, provided the original work is properly cited.

\begin{abstract}
Numerical treatments for the generalized Burger's-Huxley GBH equation are presented. The treatments are based on cardinal Chebyshev and Legendre basis functions with Galerkin method. Gauss quadrature formula and El-gendi method are used to convert the problem into a system of ordinary differential equations. The numerical results are compared with the literatures to show efficiency of the proposed methods.
\end{abstract}

\section{Introduction}

Hodgkin and Huxley [1] proposed a model in order to explain the ionic mechanisms underlying the initiation and propagation of action potentials in the squid giant axon. Satsuma investigated in 1986 that the generalized Burger's-Huxley equation $(\mathrm{GBH})$ shows a prototype model for describing the interaction between reaction mechanisms, convection effects, and diffusion transports [2]. The most general form of $\mathrm{GBH}$ is [3]

$$
\begin{array}{r}
u_{t}+\alpha u^{\delta} u_{x}-u_{x x}=\beta u\left(1-u^{\delta}\right)\left(u^{\delta}-\gamma\right), \\
a \leq x \leq b, t \geq 0,
\end{array}
$$

with the following initial and boundary conditions:

$$
\begin{gathered}
u(x, 0)=q(x), \quad a<x<b, \\
u(a, t)=f_{1}(t), \quad u(b, t)=f_{2}(t), \quad t>0,
\end{gathered}
$$

where $\alpha$ is a real parameter, $\beta \geq 0, \gamma \in(0,1)$, and $\delta>0$. If $\beta=0$, then (1) becomes the generalized Burgers' equation which is solved in [4]. For $\alpha=0$, it changes to the generalized Huxley equation [5]. Many researchers established numerical solutions of the $\mathrm{GBH}$ equation by using various numerical techniques. In [6] spectral collocation method is applied. Moreover, Darvishi's preconditionings are employed to reduce round-off error in this method. In [7] a new domain decomposition algorithm based on Chebyshev polynomials and preconditioning is presented for solving $\mathrm{GBH}$ equation. In [8] up to tenth-order finite difference schemes are proposed to solve the GBH equation. In [9] Chebyshev spectral collocation with the domain decomposition is applied to find the numerical solution of the GBH equation.

Recently, in [10], a fourth-order finite difference scheme in a two-time level recurrence relation is proposed for the numerical solution of the GBH equation. The local discontinuous Galerkin method for Burger's-Huxley equation has been studied in [11].

However, the various Galerkin algorithms have been applied in [12-14] for the numerical solutions of the ordinary differential equations. In this paper, the $\mathrm{GBH}$ equation is solved by nodal Galerkin methods. These methods have the advantages of both Galerkin and collocation methods. Collocation methods are derived from a strong form of the PDE and share many of the same advantages and disadvantages. Foremost is that they are easy to derive and to implement for a wide class of problems like constant coefficient, variable coefficient, and nonlinear problesms. Since collocation methods require the solution to satisfy the PDE at a set of grid points, they are naturally nodal approximations and will have 
aliasing errors even for constant coefficient problems. A main tradeoff is that there is little formal mathematical guidance on how to derive a stable approximation or how to implement boundary conditions. The latter makes it difficult to extend collocation methods to complex geometries or to systems of equations. Like finite difference methods, collocation methods are most easily applied to geometries that we can map onto a simple square or cube. Within those constraints, however, collocation methods will give spectrally accurate approximations.

Galerkin methods are derived from a weak form of the equations. They are less easily derived than collocation methods, but the formulation naturally leads to stable approximations and gives guidance on how to implement boundary conditions. Galerkin methods can be either nodal or modal. Modal approximations can be significantly more accurate than nodal approximations, depending on the problem. They are much harder to derive and more complex to implement, however, particularly for variable coefficient, nonlinear, or multidimensional problems.

Nodal Galerkin methods are intermediate between collocation and Galerkin methods. These methods start with the Galerkin formulation and the solutions are presented in a nodal form. Quadratures approximations are used to find the integrals that arise. The result is nodal methods which are significantly easier to implement than the Galerkin method and it can be extended to solve problems in complex geometries [15].

Furthermore, the presence of nonlinear term complicates the computation of the stiffness matrix $[15,16]$. So nodal Galerkin methods are used and results are compared with a fourth-order finite difference scheme which implies to a nonlinear system [5] but our schemes imply to linear system which is easy to solve. Also, we compare our results with a new domain decomposition algorithm based on Chebyshev polynomials and preconditioning [7]. In this study, the spectral collocation methods with the fourth-order RungeKutta method for time integration are used to solve the $\mathrm{GBH}$ equation. Moreover, preconditioning with the domain decomposition method is employed to reduce the round-off error in spectral collocation (pseudospectral) method. However, in our schemes we do not divide the domain into subdomains and we do not use any preconditioning to the resulted system. On the other hand, the domain decomposition method demanded small-step times to reach a good accuracy but in our methods we use big-step time and arrive to the same accuracy.

The remainder of this paper is organized as follows. In Section 2, we present Galerkin method with Chebyshev cardinal function as a basis function and we give the solution at the Chebyshev Gauss-Lobatto points. In Section 3, the Chebyshev Galerkin method with El-gendi quadrature is presented. In Section 4, El-gendi Legendre Galerkin method is described and we will use the Legendre cardinal function and the approximate solution will be presented at the Legendre Gauss-Lobatto points. In Section 5, numerical experiments are given and comparisons with the literatures to illustrate the efficiency of our methods are presented.

\section{Gauss Chebyshev Galerkin (GCG) Method}

In this section we explain the Gauss Chebyshev Galerkin method and illustrate how it is used to solve the problem (1) and (2) in case $a=-1$ and $b=1$. Let us now define some functional spaces. Let $\omega(x)$ be a weight function on the interval $(-1,1)$ and $L_{\omega}^{2}(-1,1)$ is the Banach space of the measurable functions $u:(-1,1) \rightarrow R$ such that

$$
\int_{-1}^{1}|u(x)|^{2} \omega(x) d x<+\infty .
$$

The space $L_{\omega}^{2}(-1,1)$ is a Hilbert space for the following inner product:

$$
(u, v)_{\omega}=\int_{-1}^{1} u(x) v(x) \omega(x) d x
$$

where

$$
\omega(x)=\frac{1}{\sqrt{x^{2}-1}} .
$$

Now, for any nonnegative integer $m$ the space $H_{\omega}^{m}(-1,1)$ is the space of all functions $h \in L_{\omega}^{2}(-1,1)$ such that the derivatives of $h$ of order up to $m$ can be represented by functions in $L_{\omega}^{2}(-1,1)$ which is associated with the following norm:

$$
\|v\|_{m, \omega}=\left(\sum_{k=0}^{m}|v|_{k, \omega}^{2}\right)^{1 / 2} .
$$

We also denote in particular the following space:

$$
H_{0, \omega}^{1}(-1,1)=\left\{u \in H_{\omega}^{1}(-1,1): u(-1, t)=u(1, t)=0\right\} .
$$

Now, Let $N$ be any positive integer and $P^{N}$ the space of polynomials of degree at most $N$; we set

$$
X^{N}=P^{N} \cap H_{0, \omega}^{1} .
$$

We started by considering the approximation:

$$
u^{N}(x, t)=\sum_{l=0}^{N} U_{l}(t) \varphi_{l}(x),
$$

where $u^{N}(x, t)$ denotes the approximate value of $u(x, t)$, $\left\{\varphi_{l}\right\}_{l=0}^{N}$ is the set of appropriate polynomials of degree $N$, and $\left\{U_{l}(t)\right\}_{l=0}^{N}$ is a set of coefficients. To have the approximation that satisfies the boundary conditions, we set $U_{0}=U_{N}=0$. Now, the weighted Galerkin method [17] takes the form: find $u^{N} \in X^{N}$

$$
\begin{gathered}
\left(u_{t}^{N}, v\right)_{\omega}-\frac{\alpha}{\delta+1}\left(\left(u^{N}\right)^{\delta+1}, v_{x}\right)_{\omega}+\left(u_{x}^{N}, v_{x}\right)_{\omega} \\
-\beta\left(\left(\left(u^{N}\right)^{\delta+1}-\left(u^{N}\right)^{2 \delta+1}, v\right)_{\omega}\right. \\
\left.-\gamma\left(u^{N}-\left(u^{N}\right)^{\delta+1}, v\right)_{\omega}\right)=0 \\
\forall v \in X^{N}, t>0 .
\end{gathered}
$$


Now we will take advantage of the flexibility given to us by the nodal representation of a polynomial. Since $u^{N}(x, t)$ satisfies (10) for any function $v$, it must satisfy the same condition for all linear combinations of the test functions:

$$
\widetilde{v}=\sum_{k=0}^{N} b_{k} v_{k}(x)
$$

where $b_{k}$ are arbitrary coefficients. Since the test function $v$ is an $\mathrm{Nth}$-order polynomial, we can write it in the following form:

$$
v=\sum_{l=0}^{N} V_{l} \varphi_{l}(x)
$$

where $\varphi_{l}(x)$ is the cardinal Chebyshev polynomial and the nodal values $V_{l}$ are arbitrary, except that $V_{0}=V_{N}=0$ to ensure that $v$ satisfies the boundary conditions. We denote the approximation of the solution at the discrete grid points by $u^{N}\left(x_{j}, t\right)$, where (1) and (2) are enforced at the collocation points $x_{j}$ where

$$
x_{j}=\cos \left(\frac{j \pi}{N}\right), \quad j=0,1, \ldots, N .
$$

Since the Chebyshev Gauss quadrature formula is given as follows:

$$
\int_{-1}^{1} \frac{f(x)}{\sqrt{1-x^{2}}} d x \approx \sum_{j=0}^{N} \omega_{j} f\left(x_{j}\right)
$$

where $\omega_{j}$ 's are given by

$$
\omega_{0}=\frac{\pi}{2 N}, \quad \omega_{j}=\frac{\pi}{N}, \quad \omega_{N}=\frac{\pi}{2 N} .
$$

In the Gauss Chebyshev Galerkin method, the trial function space coincides with the test function space $X^{N}$ which is a finite dimensional subspace of $H_{\omega, 0}^{1}(-1,1)$ and $X^{N}$ is given as follows:

$$
X^{N}=\operatorname{span}\left\{\varphi_{1}(x), \varphi_{2}(x), \ldots, \varphi_{N-1}(x)\right\},
$$

whereas $\varphi_{j}(x)$ is given by

$$
\varphi_{j}(x)=\frac{2 \theta_{j}}{N} \sum_{k=0}^{N} \theta_{k} T_{k}\left(x_{j}\right) T_{n}(x), \quad j=0,1, \ldots, N
$$

for all $\theta_{k}=1$, except $\theta_{0}=\theta_{N}=1 / 2$ and

$$
\varphi_{j}\left(x_{k}\right)= \begin{cases}0 & \text { if } j \neq k \\ 1 & \text { if } j=k\end{cases}
$$

The grid points $x_{k}$ are called Chebyshev Gauss-Lobatto points, which are the extremal points of the Chebyshev polynomial $T_{N}(x)$. To get the nodal Galerkin approximation, we replace the integrals in (10) by Chebyshev Gauss-Lobatto quadrature.
Then the first discrete inner product becomes

$$
\left(u_{t}^{N}, v\right)_{\omega}=\sum_{j=0}^{N} \omega_{j}\left(\sum_{n=0}^{N} \dot{U}_{n} \varphi_{n}\left(x_{j}\right) \sum_{m=0}^{N} V_{m} \varphi_{m}\left(x_{j}\right)\right) .
$$

since $\varphi_{i}\left(x_{j}\right)=\delta_{i j}$, then the sum reduces to

$$
\left(u_{t}^{N}, v\right)_{\omega}=\sum_{j=0}^{N} \omega_{j} \dot{U}_{j} V_{j}
$$

and the second inner product is

$$
\begin{aligned}
\left(\left(u^{N}\right)^{\delta+1}, v_{x}\right)_{\omega} & =\sum_{j=0}^{N} \omega_{j}\left(u_{j}^{N}\right)^{\delta+1} \sum_{m=0}^{N} V_{m} \varphi_{m}^{\prime}\left(x_{j}\right) \\
& =\sum_{m=0}^{N} V_{m}\left(\left.\sum_{j=0}^{N} \omega_{j}\left[\left(u^{N}\right)^{\delta+1} \varphi_{m}^{\prime}(x)\right]\right|_{x=x_{j}}\right) .
\end{aligned}
$$

If we rename the indices $m \leftarrow j$ and $j \leftarrow k$, then we have

$$
\left(\left(u^{N}\right)^{\delta+1}, v_{x}\right)_{\omega}=\sum_{j=0}^{N} V_{j}\left(\left.\sum_{k=0}^{N} \omega_{k}\left[\left(u^{N}\right)^{\delta+1} \varphi_{j}^{\prime}(x)\right]\right|_{x=x_{k}}\right),
$$

where the first derivative of the cardinal functions $\varphi_{j}(x)$ at the points $x_{l}$ have the entries of the differentiation matrix [18]:

$$
\begin{array}{r}
\varphi_{j}^{\prime}\left(x_{l}\right)=\frac{4 \theta_{j}}{N} \sum_{k=1}^{N} \sum_{\substack{n=0 \\
(n+k) \text { odd }}}^{k-1} \frac{\theta_{k} k}{c_{n}} T_{k}\left(x_{j}\right) T_{n}\left(x_{l}\right), \\
j=0,1, \ldots, N ;
\end{array}
$$

the second derivatives are

$$
\begin{array}{r}
\varphi_{j}^{\prime \prime}\left(x_{l}\right)=\frac{4 \theta_{j}}{N} \sum_{k=2}^{N} \sum_{\substack{n=0 \\
(n+k) \text { even }}}^{k-2} \frac{\theta_{k} k\left(k^{2}-n^{2}\right)}{c_{n}} T_{k}\left(x_{j}\right) T_{n}\left(x_{l}\right), \\
j=0,1, \ldots, N .
\end{array}
$$

In the same way, we can find the third and fourth term in

$$
\begin{gathered}
\left(u_{x}^{N}, v_{x}\right)_{\omega}=\sum_{j=0}^{N} V_{j}\left(\left.\sum_{k=0}^{N} \omega_{k}\left[u_{x}^{N} \varphi_{j}^{\prime}(x)\right]\right|_{x=x_{k}}\right) \\
\left(\left(u^{N}\right)^{\delta+1}-\left(u^{N}\right)^{2 \delta+1}, v\right)_{\omega} \\
=\left.\sum_{j=0}^{N} \omega_{j}\left[\left(u^{N}\right)^{\delta+1}-\left(u^{N}\right)^{2 \delta+1}\right]\right|_{x=x_{j}} V_{j}, \\
\left(u^{N}-\left(u^{N}\right)^{\delta+1}, v\right)_{\omega}=\left.\sum_{j=0}^{N} \omega_{j}\left[u^{N}-\left(u^{N}\right)^{\delta+1}\right]\right|_{x=x_{j}} V_{j} .
\end{gathered}
$$


By using (20), (22), (23), (24), and (25), we can write the discrete weak form in the following form:

$$
\begin{gathered}
\sum_{j=0}^{N} V_{j}\left[\omega_{j} \dot{U}_{j}-\left.\frac{\alpha}{\delta+1} \sum_{k=0}^{N} \omega_{k}\left[\left(u^{N}\right)^{\delta+1} \varphi_{j}^{\prime}(x)\right]\right|_{x=x_{k}}\right. \\
+\left.\sum_{k=0}^{N} \omega_{k}\left[u_{x}^{N} \varphi_{j}^{\prime}(x)\right]\right|_{x=x_{k}} \\
-\beta \omega_{j}\left(\left.\left[\left(u^{N}\right)^{\delta+1}-\left(u^{N}\right)^{2 \delta+1}\right]\right|_{x=x_{j}}\right. \\
\left.\left.-\left.\gamma\left[u^{N}-\left(u^{N}\right)^{\delta+1}\right]\right|_{x=x_{j}}\right)\right]=0 .
\end{gathered}
$$

Since $V_{j}$ 's are linearly independent, the coefficient of each $V_{j}$ must be zero, so

$$
\begin{gathered}
\omega_{j} \dot{U}_{j}-\left.\frac{\alpha}{\delta+1} \sum_{k=0}^{N} \omega_{k}\left[\left(u^{N}\right)^{\delta+1} \varphi_{j}^{\prime}(x)\right]\right|_{x=x_{k}} \\
\left.+\left.\sum_{k=0}^{N} \omega_{k}\left[u_{x}^{N} \varphi_{j}^{\prime}(x)\right]\right|_{x=x_{k}}\right]\left.\right|_{x=x_{j}} \\
-\beta \omega_{j}\left(\left[\left(u^{N}\right)^{\delta+1}-\left(u^{N}\right)^{2 \delta+1}\right]=0,\right. \\
\left.-\left.\gamma\left[u^{N}-\left(u^{N}\right)^{\delta+1}\right]\right|_{x=x_{j}}\right)=1, \ldots, N-1 .
\end{gathered}
$$

Notice that the end points, $j=0$ and $j=N$, are not included, since $V_{0}=V_{N}=0$ satisfying the boundary conditions. We specify the unknowns at those points by the boundary conditions. We complete the approximation (27) by using the approximation (9). So we have

$$
\begin{aligned}
& \omega_{j} \dot{U}_{j}= \frac{\alpha}{\delta+1} \sum_{l=0}^{N} C_{j l} U_{l}^{\delta+1}-\sum_{l=0}^{N} B_{j l} U_{l} \\
&+\beta \omega_{j}\left(\left[U_{j}^{\delta+1}-U_{j}^{2 \delta+1}\right]-\gamma\left[U_{j}-U_{j}^{\delta+1}\right]\right), \\
& \quad j=1, \ldots, N-1, U_{0}=U_{N}=0,
\end{aligned}
$$

where

$$
B_{j l}=\sum_{k=0}^{N} \omega_{k} \varphi_{l}^{\prime}\left(x_{k}\right) \varphi_{j}^{\prime}\left(x_{k}\right), \quad C_{j l}=\omega_{l} \varphi_{j}^{\prime}\left(x_{l}\right) .
$$

The resulted system of ODEs has been solved by using fourthorder Runge-Kutta solver.

\section{El-Gendi Chebyshev Galerkin (ECG) Method}

In this method, the trial and test spaces are identical, so that we define for $m \geq 0$ the space $H^{m}(-1,1)$ to be a vector space of functions $v \in L^{2}(-1,1)$ such that all distributional derivatives of $v$ of order up to $m$ can be represented by functions in $L^{2}(-1,1)$ which is a Hilbert space for the inner product:

$$
(u, v)=\int_{-1}^{1} u v d x
$$

Since the functions of $H^{1}(-1,1)$ are continuous up to the boundary by Sobolev imbedding theorem, it is meaningful. So, the solution subspace of $H^{1}(-1,1)$ is given as follows:

$$
H_{0}^{1}(-1,1)=\left\{u \in H^{1}(-1,1): u(-1, t)=u(1, t)=0\right\},
$$

which is a Hilbert space for the inner product defined as follows:

$$
(u, v)=\int_{-1}^{1} u_{x} v_{x} d x
$$

where $f_{x}=d f / d x$.

The weak forms of (1) and (2) are given by the following: find $u \in H_{0}^{1}(-1,1)$ such that

$$
\begin{array}{r}
\int_{-1}^{1} \frac{\partial u}{\partial t} v d x-\frac{\alpha}{\delta+1} \int_{-1}^{1} u^{(\delta+1)} \frac{\partial v}{\partial x} d x \\
+\int_{-1}^{1} \frac{\partial u}{\partial x} \frac{\partial v}{\partial x} d x-\beta \int_{-1}^{1} u\left(1-u^{\delta}\right)\left(u^{\delta}-\gamma\right) v d x=0 \\
\forall v \in H_{0}^{1}(-1,1), t>0 .
\end{array}
$$

In this section, El-gendi formula has been used as follow: Let

$$
\int_{-1}^{1} g(x) d x \cong \sum_{k=0}^{N} b_{N k} g\left(x_{k}\right)
$$

where $b_{N k}$ are given by [19]:

$$
\begin{gathered}
b_{N k}=\frac{4}{N} \sum_{j=0}^{N / 2} \frac{\theta_{s}}{4 j^{2}-1} \cos \frac{2 k \pi j}{N}, \quad k=1,2, \ldots, N-1, \\
b_{N 0}=b_{N N}=\frac{1}{N^{2}-1} .
\end{gathered}
$$

In this case we use the following space:

$$
\widetilde{X}^{N}=H_{0}^{1}(-1,1) \cap P^{N} .
$$

Now the discrete weak form is given as follows: find $u^{N} \in \widehat{X}^{N}$

$$
\begin{aligned}
& \left(u_{t}^{N}, v\right)_{N}-\frac{\alpha}{\delta+1}\left(\left(u^{N}\right)^{\delta+1}, v_{x}\right)_{N}+\left(u_{x}^{N}, v_{x}\right)_{N} \\
& -\beta\left(\left(\left(u^{N}\right)^{\delta+1}-\left(u^{N}\right)^{2 \delta+1}, v\right)_{N}\right. \\
& \left.-\gamma\left(u^{N}-\left(u^{N}\right)^{\delta+1}, v\right)_{N}\right)=0 \quad \forall v \in \widehat{X}^{N}, t>0,
\end{aligned}
$$


where

$$
(f, g)_{N}=\sum_{j=0}^{N} b_{N j} f\left(y_{j}\right) g\left(y_{j}\right)
$$

Then the first term is given as follows:

$$
\left(u_{t}^{N}, v\right)_{N}=\sum_{j=0}^{N} b_{N j} \dot{U}_{j} V_{j} .
$$

Similarly as above we can evaluated the rest of terms in the discrete weak form and we can write the resulting system after some manipulations as follows:

$$
\begin{aligned}
& b_{N j} \dot{U}_{j}= \frac{\alpha}{\delta+1} \sum_{l=0}^{N} \widetilde{C}_{j l} U_{l}^{\delta+1}-\sum_{l=0}^{N} \widetilde{B}_{j l} U_{l} \\
&+\beta b_{N j}\left(\left[U_{j}^{\delta+1}-U_{j}^{2 \delta+1}\right]-\gamma\left[U_{j}-U_{j}^{\delta+1}\right]\right), \\
& \quad j=1, \ldots, N-1, U_{0}=U_{N}=0,
\end{aligned}
$$

where

$$
\widetilde{B}_{j l}=\sum_{k=0}^{N} b_{N k} \varphi_{l}^{\prime}\left(x_{k}\right) \varphi_{j}^{\prime}\left(x_{k}\right), \quad \widetilde{C}_{j l}=b_{N l} \varphi_{j}^{\prime}\left(x_{l}\right) .
$$

The resulted system of ODEs has been solved by using fourthorder Runge-Kutta solver.

\section{El-Gendi Legendre Galerkin (ELG) Method}

Analogous to the previous section we consider the Legendre cardinal function based on Legendre Gauss-Lobatto (LGL) nodes. El-gendi approximation will be used with a linear combination of the Legendre cardinal function as follows [20]:

$$
\begin{array}{r}
\psi_{j}(y)=\frac{1}{N(N-1)} \sum_{k=0}^{N}(2 k+1) \frac{L_{k}\left(y_{j}\right) L_{k}(y)}{\left[L_{N-1}\left(y_{j}\right)\right]^{2}}, \\
j=0,1, \ldots, N,
\end{array}
$$

where $y_{j}$ are the Legendre Gauss-Lobatto points and $\psi_{j}(y)$ satisfies the condition:

$$
\psi_{j}\left(y_{k}\right)= \begin{cases}1, & \text { if } j=k, \\ 0, & \text { if } j \neq k\end{cases}
$$

Now, the discrete weak form in the case of Legendre Galerkin method is as follows: find $u^{N} \in \widehat{X}^{N}\left(u_{t}^{N}, v\right)_{N}-(\alpha /(\delta+1))$

$$
\begin{aligned}
& \left(\left(u^{N}\right)^{\delta+1}, v_{x}\right)_{N}+\left(u_{x}^{N}, v_{x}\right)_{N} \\
& -\beta\left(\left(\left(u^{N}\right)^{\delta+1}-\left(u^{N}\right)^{2 \delta+1}, v\right)_{N}\right. \\
& \left.\quad-\gamma\left(u^{N}-\left(u^{N}\right)^{\delta+1}, v\right)_{N}\right)=0, \quad \forall v \in \widehat{X}^{N}, t>0,
\end{aligned}
$$

where

$$
(f, g)_{N}=\sum_{j=0}^{N} b_{N j} f\left(y_{j}\right) g\left(y_{j}\right) \text {. }
$$

Then the first discrete inner product becomes

$$
\left(u_{t}^{N}, v\right)_{N}=\sum_{j=0}^{N} b_{N j}\left(\sum_{n=0}^{N} \dot{U}_{n} \psi_{n}\left(y_{j}\right) \sum_{m=0}^{N} V_{m} \psi_{m}\left(y_{j}\right)\right) \text {. }
$$

Since $\psi_{i}\left(y_{j}\right)=\delta_{i j}$, then the sum reduces to

$$
\left(u_{t}^{N}, v\right)_{N}=\sum_{j=0}^{N} b_{N j} \dot{U}_{j} V_{j},
$$

and the second inner product is

$$
\begin{aligned}
\left(\left(u^{N}\right)^{\delta+1}, v_{x}\right)_{N} & =\sum_{j=0}^{N} b_{N j}\left(u_{j}^{N}\right)^{\delta+1} \sum_{m=0}^{N} V_{m} \psi_{m}^{\prime}\left(y_{j}\right) \\
& =\sum_{m=0}^{N} V_{m}\left(\left.\sum_{j=0}^{N} b_{N j}\left[\left(u^{N}\right)^{\delta+1} \psi_{m}^{\prime}(y)\right]\right|_{y=y_{j}}\right) .
\end{aligned}
$$

If we rename the indices $m \leftarrow j$ and $j \leftarrow k$, then we have

$$
\left(\left(u^{N}\right)^{\delta+1}, v_{x}\right)_{N}=\sum_{j=0}^{N} V_{j}\left(\left.\sum_{k=0}^{N} b_{N k}\left[\left(u^{N}\right)^{\delta+1} \psi_{j}^{\prime}(y)\right]\right|_{y=y_{k}}\right),
$$

where $\left.\left(\psi_{j}^{\prime}\right)\right|_{y=y_{l}}$ is the first-order differentiation matrix that depends on Legendre polynomial at the LGL nodes and has the entries given by [21]:

$$
\left.\left(\psi_{j}^{\prime}\right)\right|_{y=y_{l}}=\sum_{j=m}^{N} \sum_{k=0}^{[(j-1) / 2]} \frac{2 j+1}{N(N-1)} \frac{L_{j}\left(y_{i}\right)}{\left[L_{N-1}\left(y_{i}\right)\right]^{2}} a_{k, 1}^{(j)} y_{l}^{j-2 k-1},
$$

where

$$
a_{k, 1}^{(j)}=\frac{(-1)^{k}(2 j-2 k) !(j-2 k) !}{2^{j}(j-k) !(j-2 k) ! k !} .
$$

Also, $\left.\left(\psi_{j}^{\prime \prime}\right)\right|_{y=y_{l}}$ has the following formula:

$$
\left.\left(\psi_{j}^{\prime \prime}\right)\right|_{y=y_{l}}=\sum_{j=m}^{N} \sum_{k=0}^{[(j-2) / 2]} \frac{2 j+1}{N(N-1)} \frac{L_{j}\left(y_{i}\right)}{\left[L_{N-1}\left(y_{i}\right)\right]^{2}} a_{k, 2}^{(j)} y_{l}^{j-2 k-2},
$$

where

$$
a_{k, 2}^{(j)}=\frac{(-1)^{k}(2 j-2 k) !(j-2 k) !(j-2 k-1) !}{2^{j}(j-k) !(j-2 k) ! k !} .
$$


Then by using equations (45), (47), (49), (50), and (52) we can evaluate all the terms in the weak form () and we arrive to the following system:

$$
\begin{aligned}
b_{N j} \dot{U}_{j}= & \frac{\alpha}{\delta+1} \sum_{l=0}^{N} \widehat{C}_{j l} U_{l}^{\delta+1}-\sum_{l=0}^{N} \widehat{B}_{j l} U_{l} \\
& +\beta b_{N j}\left(\left[U_{j}^{\delta+1}-U_{j}^{2 \delta+1}\right]-\gamma\left[U_{j}-U_{j}^{\delta+1}\right]\right), \\
& \quad j=1, \ldots, N-1, U_{0}=U_{N}=0,
\end{aligned}
$$

where

$$
\widehat{B}_{j l}=\sum_{k=0}^{N} b_{N k} \psi_{l}^{\prime}\left(y_{k}\right) \psi_{j}^{\prime}\left(y_{k}\right), \quad \widehat{C}_{j l}=b_{N l} \psi_{j}^{\prime}\left(y_{l}\right) .
$$

As before, we use the fourth-order Runge-Kutta method to solve the resulted system (54).

Remark. In case of nonhomogenous boundary conditions the trial and test function space are the Sobolev spaces. So the boundary conditions are accounted naturally in the weak formulation. Moreover, the finite dimensional subspaces of the Sobolev spaces will be the whole space of polynomials. On the other hand, the methods which depend on numerical integration enforce the boundary condition explicitly or enforce the boundary points a particular linear combination of the approximate equation and the boundary condition; see $[15$, page $130-131]$ for more details.

\section{Numerical Experiments}

In this section we will give two examples and we will use MATLAB 7.0 software to obtain the numerical results. Consider the $\mathrm{GBH}$ equation:

$$
u_{t}+\alpha u^{\delta} u_{x}-u_{x x}=\beta u\left(1-u^{\delta}\right)\left(u^{\delta}-\gamma\right) ; \quad a \leq x \leq b, t \geq 0,
$$

where $\alpha, \beta, \delta$, and $\gamma$ are arbitrary constants and $a$ and $b$ are real numbers.

The initial condition is

$$
u(x, 0)=\left[\frac{\gamma}{2}+\frac{\gamma}{2} \tanh \left(\eta_{1} x\right)\right]^{1 / \delta}
$$

where

$$
\eta_{1}=\frac{-\alpha \delta+\delta \sqrt{\alpha^{2}+4 \beta(1+\delta)}}{4(1+\delta)} \gamma
$$

The boundary conditions are

$$
\begin{aligned}
& u(a, t)=\left[\frac{\gamma}{2}+\frac{\gamma}{2} \tanh \left[\eta_{1}\left(a-\eta_{2} t\right)\right]\right]^{1 / \delta}, \quad t \geq 0 \\
& u(b, t)=\left[\frac{\gamma}{2}+\frac{\gamma}{2} \tanh \left[\eta_{1}\left(b-\eta_{2} t\right)\right]\right]^{1 / \delta}, \quad t \geq 0
\end{aligned}
$$

where

$$
\eta_{2}=\frac{\gamma \alpha}{1+\delta}-\frac{(1+\delta-\gamma)\left(-\alpha+\sqrt{\alpha^{2}+4 \beta(1+\delta)}\right)}{2(1+\delta)},
$$

and the exact solution is

$$
u(x, t)=\left[\frac{\gamma}{2}+\frac{\gamma}{2} \tanh \left[\eta_{1}\left(x-\eta_{2} t\right)\right]\right]^{1 / \delta}
$$

The following error notations are defined:

$$
\begin{gathered}
\text { abser }=\left|u_{\mathrm{ex}}\left(x_{i}\right)-u_{\mathrm{app}}\left(x_{i}\right)\right|, \quad i=1, \ldots, N-1, \\
\text { Maxer }=\max _{i}\left|u_{\mathrm{ex}}\left(x_{i}\right)-u_{\mathrm{app}}\left(x_{i}\right)\right|, \quad i=1, \ldots, N-1,
\end{gathered}
$$

where $u_{\text {ex }}\left(x_{i}\right)$ and $u_{\text {app }}\left(x_{i}\right)$ are the exact and approximate solutions, respectively. Since the Gauss-Lobatto points are in interval $[-1,1]$, the interval $[a, b]$ is mapped to $[-1,1]$ by a linear mapping defined by

$$
X_{i}=\left(\frac{b-a}{2}\right) \xi_{i}+\left(\frac{b+a}{2}\right), \quad i=0, \ldots, N,
$$

where $\xi_{i}$ are the Gauss-Lobatto nodes.

Example 1. We consider the Burger's-Huxley equation (1) with $\alpha=1, \beta=1, \gamma=0.001, \Delta t=0.0001, a=0, b=1$, and $N=16$. In Table 1, we compare the maximum error obtained by our method and domain decomposition algorithm in [7]. As can be seen from this table, our methods are more accurate in time $t=0.2$.

To show the solitary wave evolution with time, we expand the computation domain to $\left[-10^{4}, 10^{4}\right]$ and we plot the numerical solution and exact solution in Figure 1 for the values $\alpha=1, \beta=1, \gamma=0.001$, and $\Delta t=0.0001$ at $t \in[0,10]$. In Figure 2, we expand the computation domain to $[-10,20]$ and plot the numerical solution and exact solution for the values $\alpha=1, \beta=1$, and $\gamma=2$.

Example 2. In Table 2, the problem solved for various $\beta, \gamma$ and for $\Delta t=0.1, \alpha=5, \delta=1, a=0, b=1, N=16$, and $T=0.30$.

From Table 2 it is deduced that the proposed methods give more accurate results to those in [7] and the time step is not small as in [7]. Also, it is noted that the accuracy decreased when $\beta$ increased and the accuracy increased when $\gamma$ decreased.

Example 3. In Table 3, the problem solved for various $\delta=1$ and for $\Delta t=0.01, \alpha=0, \gamma=0.001, \delta=1, \beta=1, a=0$, $b=1$, and $N=16$. In Table 3 , we show maximum error and compare with the corresponding results in [10].

Example 4. In Table 4, we will solve the same problem but with $\delta=2$. 
TABLE 1

\begin{tabular}{lllllr}
\hline$T$ & $\delta$ & Maxer. GCG & Maxer. ECG & Maxer. ELG & Maxer. [7] \\
\hline \multirow{2}{*}{0.2} & $\delta=1$ & $3.9133 E-08$ & $3.6586 E-08$ & $3.7213 E-08$ & $1.0138 E-08$ \\
& $\delta=4$ & $1.2797 E-05$ & $1.3119 E-05$ & $1.2613 E-05$ & $1.3139 E-05$ \\
& $\delta=8$ & $3.5503 E-05$ & $3.4622 E-05$ & $3.4999 E-05$ & $3.5540 E-05$ \\
\hline \multirow{2}{*}{1.0} & $\delta=1$ & $1.9536 E-07$ & $1.8791 E-07$ & $1.8703 E-07$ & $4.6848 E-08$ \\
& $\delta=4$ & $6.3927 E-05$ & $6.1823 E-05$ & $6.1625 E-05$ & $1.5325 E-05$ \\
& $\delta=8$ & $1.7284 E-04$ & $1.6850 E-04$ & $1.6606 E-04$ & $4.1406 E-05$ \\
\hline
\end{tabular}

TABLE 2

\begin{tabular}{cccccc}
\hline$\gamma$ & $\beta$ & Maxer. GCG & Maxer. ECG & Maxer. ELG & Maxer. [7] \\
\hline \multirow{3}{*}{$10^{-3}$} & 1 & $5.6588 E-09$ & $5.6487 E-09$ & $5.5590 E-09$ & $3.1616 E-08$ \\
& 10 & $4.0136 E-08$ & $3.9438 E-08$ & $3.5810 E-08$ & $3.9742 E-07$ \\
& 100 & $1.5500 E-06$ & $1.5469 E-06$ & $1.5425 E-06$ & $5.0365 E-06$ \\
\hline \multirow{3}{*}{$10^{-4}$} & 1 & $5.6218 E-11$ & $5.6246 E-11$ & $5.2789 E-11$ & $3.1630 E-10$ \\
& 10 & $3.9544 E-10$ & $3.9474 E-10$ & $3.7009 E-10$ & $3.9760 E-09$ \\
& 100 & $1.5484 E-08$ & $1.5480 E-08$ & $1.5369 E-08$ & $3.1632 E-12$ \\
$10^{-5}$ & 1 & $5.6219 E-13$ & $5.6222 E-13$ & $5.2509 E-13$ & $3.9762 E-11$ \\
& 10 & $3.9485 E-12$ & $3.9478 E-12$ & $3.6859 E-12$ & $5.0392 E-10$ \\
\hline
\end{tabular}

TABLE 3

\begin{tabular}{cccccc}
\hline$T$ & $X$ & abser. GCG & abser. ECG & abser. ELG & abser. [10] \\
\hline \multirow{3}{*}{0.05} & 0.1 & $1.0698 E-08$ & $1.0683 E-08$ & $9.2752 E-09$ & $2.4987 E-08$ \\
& 0.5 & $9.2595 E-09$ & $9.2595 E-09$ & $9.2595 E-09$ & $2.4987 E-08$ \\
& 0.9 & $7.8921 E-09$ & $7.8701 E-09$ & $9.2845 E-09$ & $2.4987 E-08$ \\
\hline \multirow{3}{*}{0.10} & 0.1 & $2.3188 E-08$ & $2.3188 E-08$ & $2.3173 E-08$ & $4.9950 E-08$ \\
& 0.5 & $2.1749 E-08$ & $2.1748 E-08$ & $2.1749 E-08$ & $4.9950 E-08$ \\
& 0.9 & $2.0382 E-08$ & $2.0381 E-08$ & $2.0360 E-08$ & $4.9950 E-08$ \\
\hline \multirow{3}{*}{1.00} & 0.1 & $2.4872 E-07$ & $2.4870 E-07$ & $2.4729 E-07$ & $4.9950 E-07$ \\
& 0.5 & $2.4728 E-07$ & $2.4728 E-07$ & $2.4728 E-07$ & $4.9949 E-07$ \\
& 0.9 & $2.4591 E-07$ & $2.4585 E-07$ & $2.4530 E-07$ & $492-07$ \\
\hline
\end{tabular}

TABLE 4

\begin{tabular}{llcccc}
\hline$T$ & $X$ & abser. GCG & abser. ECG & abser. ELG & abser. [10] \\
\hline \multirow{3}{*}{0.05} & 0.1 & $4.8110 E-07$ & $4.5567 E-07$ & $4.2020 E-07$ & $1.1176 E-06$ \\
& 0.5 & $3.9966 E-07$ & $3.9966 E-07$ & $3.9966 E-07$ & $1.1175 E-06$ \\
& 0.9 & $3.9240 E-07$ & $3.5649 E-07$ & $4.3297 E-07$ & $1.1173 E-06$ \\
\hline \multirow{3}{*}{0.10} & 0.1 & $1.0397 E-06$ & $1.0142 E-06$ & $9.7883 E-07$ & $2.2352 E-06$ \\
& 0.5 & $9.5823 E-07$ & $9.5822 E-07$ & $9.5823 E-07$ & $2.2350 E-06$ \\
& 0.9 & $9.5091 E-07$ & $9.1499 E-07$ & $9.9147 E-07$ & $2.2347 E-06$ \\
\hline \multirow{3}{*}{1.00} & 0.1 & $1.1021 E-05$ & $1.1105 E-05$ & $1.1008 E-05$ & $2.2352 E-05$ \\
& 0.5 & $1.1057 E-05$ & $1.1056-05$ & $1.095-05$ & $2.2350 E-05$ \\
& 0.9 & $1.0841 E-05$ & $1.0995 E-05$ & & $2.2347 E-05$ \\
\hline
\end{tabular}

Example 5. In Table 5, the problem solved for $\Delta t=0.0001$, $\alpha=0.1, \beta=0.001, \gamma=0.0001, a=0, b=1, T=0.20, N=8$, and for various $\delta$.

In [7] the time step is 0.00001 which is very small and in our methods the time step is bigger than the time step in [7] so we reach the demanded order of accuracy faster than the method in [7].

\section{Conclusion}

In this paper, three efficient methods that depend on Galerkin method are used in space and a fourth-order Runge-Kutta method in time has been proposed for the generalized Burger's-Huxley equation, with high convergence. Comparisons of the computed results with exact solutions showed that the method has the capability of solving the generalized 


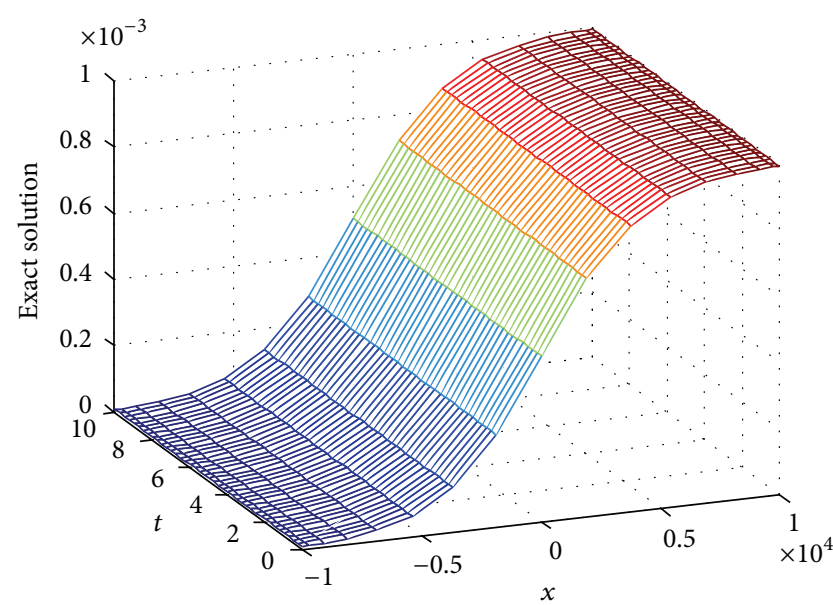

(a)

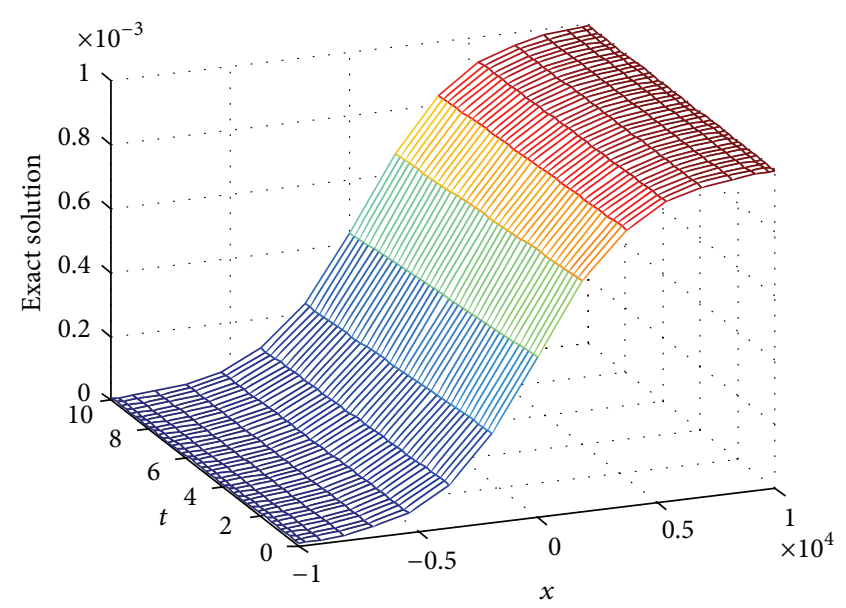

(b)

FIGURE 1: The solution at $\alpha=1, \beta=1, \gamma=0.001, a=-10^{4}, b=10^{4}$, and $t \in[0,10]$. In (a) the surface shows the exact solution and in (b) the surface shows the approximate solution.

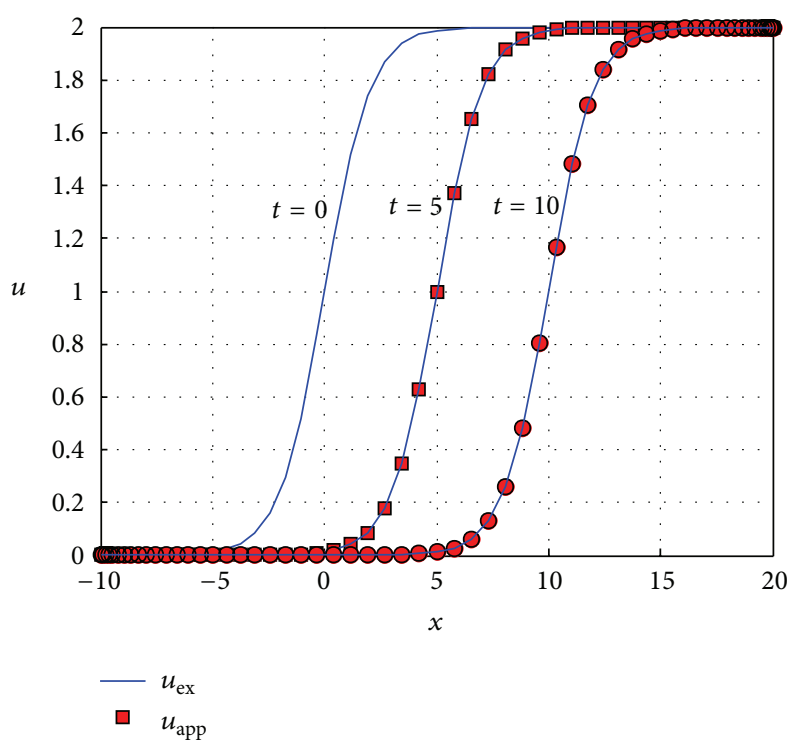

Figure 2: The solution at $\alpha=1, \beta=1, \gamma=2, a=-10, b=20$, and $N=60$.

TABLE 5

\begin{tabular}{ccccc}
\hline$\delta$ & Maxer. GCG & Maxer. ECG & Maxer. ELG & Maxer. [7] \\
\hline 1 & $5.7534 E-13$ & $3.3288 E-13$ & $2.8646 E-13$ & $3.0689 E-13$ \\
4 & $7.0834 E-10$ & $5.6124 E-10$ & $5.3350 E-10$ & $5.7108 E-10$ \\
8 & $2.2678 E-09$ & $2.0243 E-09$ & $1.9793 E-09$ & $2.1186 E-09$ \\
\hline
\end{tabular}

Burger's-Huxley equation and is also capable of producing highly accurate solutions with minimal computational effort for both time and space. It was seen that the nodal Galerkin technique approximates the exact solution very well. Since the scheme is explicit, linearization is not needed. No requiring extra effort to deal with nonlinear terms,so it is easy in use. For concrete problems where an exact solution does not exist, the present method is a very good choice to achieve a high degree of accuracy while dealing with the problems.

\section{References}

[1] A. L. Hodgkin and A. F. Huxley, "A quantitative description of Ion currents and Its applications to conduction and excitation in nerve," The Journal of Physiology, vol. 117, no. 4, pp. 500-544, 1952.

[2] J. Satsuma, M. Ablowitz, B. Fuchssteiner, and M. Kruskal, Topics in Soliton Theory and Exactly Solvable Nonlinear Equations, World Scientific, Singapore, 1987.

[3] X. Y. Wang, Z. S. Zhu, and Y. K. Lu, "Solitary wave solutions of the generalised Burgers-Huxley equation," Journal of Physics A, vol. 23, no. 3, pp. 271-274, 1990.

[4] H. M. El-Hawary and E. O. Abdel-Rahman, "Numerical solution of the generalized Burger's equation via spectral/spline methods," Applied Mathematics and Computation, vol. 170, no. 1, pp. 267-279, 2005.

[5] B. Batiha, M. S. M. Noorani, and I. Hashim, "Numerical simulation of the generalized Huxley equation by He's variational iteration method," Applied Mathematics and Computation, vol. 186, no. 2, pp. 1322-1325, 2007.

[6] M. T. Darvishi, S. Kheybari, and F. Khani, "Spectral collocation method and Darvishi's preconditionings to solve the generalized Burgers-Huxley equation," Communications in Nonlinear Science and Numerical Simulation, vol. 13, no. 10, pp. 2091-2103, 2008.

[7] M. Javidi and A. Golbabai, "A new domain decomposition algorithm for generalized Burger's-Huxley equation based on Chebyshev polynomials and preconditioning," Chaos, Solitons \& Fractals, vol. 39, no. 2, pp. 849-857, 2009.

[8] M. Sari, G. Gürarslan, and A. Zeytinoğlu, "High-order finite difference schemes for numerical solutions of the generalized Burgers-Huxley equation," Numerical Methods for Partial Differential Equations, vol. 27, no. 5, pp. 1313-1326, 2010.

[9] M. Javidi, "A modified Chebyshev pseudospectral DD algorithm for the GBH equation," Computers \& Mathematics with Applications, vol. 62, no. 9, pp. 3366-3377, 2011. 
[10] A. Bratsos, "A fourth order improved numerical scheme for the generalized Burger's-Huxley equation," American Journal of Computational Mathematics, vol. 1, no. 3, pp. 152-158, 2011.

[11] R. Zhang, X. Yu, and G. Zhao, "The local discontinuous Galerkin method for Burger's-Huxley and Burger's-Fisher equations," Applied Mathematics and Computation, vol. 218, no. 17, pp. 8773-8778, 2012.

[12] E. H. Doha and W. M. Abd-Elhameed, "Efficient spectral ultraspherical-dual-Petrov-Galerkin algorithms for the direct solution of $(2 n+1)$ th-order linear differential equations," Mathematics and Computers in Simulation, vol. 79, no. 11, pp. 3221-3242, 2009.

[13] E. H. Doha, W. M. Abd-Elhameed, and A. H. Bhrawy, "Efficient spectral ultraspherical-Galerkin algorithms for the direct solution of $2 n$ th-order linear differential equations," Applied Mathematical Modelling, vol. 33, no. 4, pp. 1982-1996, 2009.

[14] E. H. Doha and A. H. Bhrawy, "Efficient spectral-Galerkin algorithms for direct solution of fourth-order differential equations using Jacobi polynomials," Applied Numerical Mathematics, vol. 58, no. 8, pp. 1224-1244, 2008.

[15] C. Canuto, M. Y. Hussaini, A. Quarteroni, and T. A. Zang, Spectral Methods Fundamentals in Single Domains, Springer, Berlin, Germany, 2006.

[16] J. Hesthaven, S. Gottlieb, and D. Gottlieb, Spectral Methods for Time-Dependent Problems, The Cambridge Monographs on Applied and Computational Mathematics, Cambridge University Press, 2007.

[17] J. Shen, "Stable and efficient spectral methods in unbounded domains using Laguerre functions," SIAM Journal on Numerical Analysis, vol. 38, no. 4, pp. 1113-1133, 2000.

[18] E. M. E. Elbarbary and S. M. El-Sayed, "Higher order pseudospectral differentiation matrices," Applied Numerical Mathematics, vol. 55, no. 4, pp. 425-438, 2005.

[19] S. E. El-gendi, "Chebyshev solution of differential, integral and integro-differential equations," The Computer Journal, vol. 12, no. 3, pp. 282-287, 1969.

[20] M. El-Kady and M. Biomy, "Efficient Legendre pseudospectral method for solving integral and integro-differential equations," Communications in Nonlinear Science and Numerical Simulation, vol. 15, no. 7, pp. 1724-1739, 2010.

[21] M. El-Kady and M. Biomy, "Interactive Chebyshev-Legendre algorithm for linear quadratic optimal regulator systems," International Journal of Wavelets, Multiresolution and Information Processing, vol. 9, no. 3, pp. 459-483, 2011. 


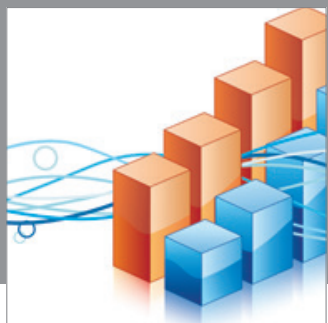

Advances in

Operations Research

mansans

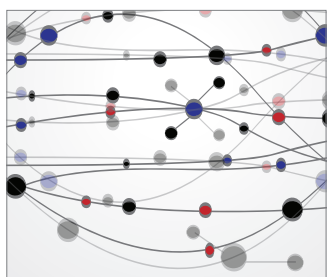

The Scientific World Journal
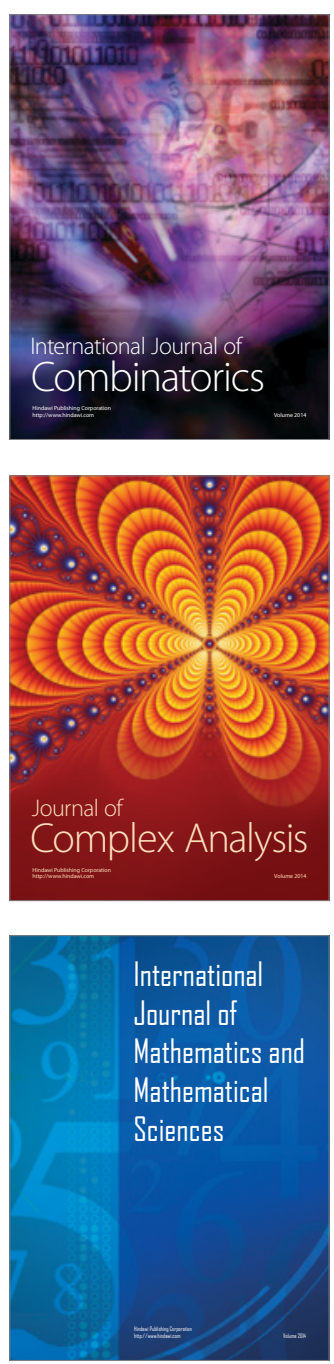
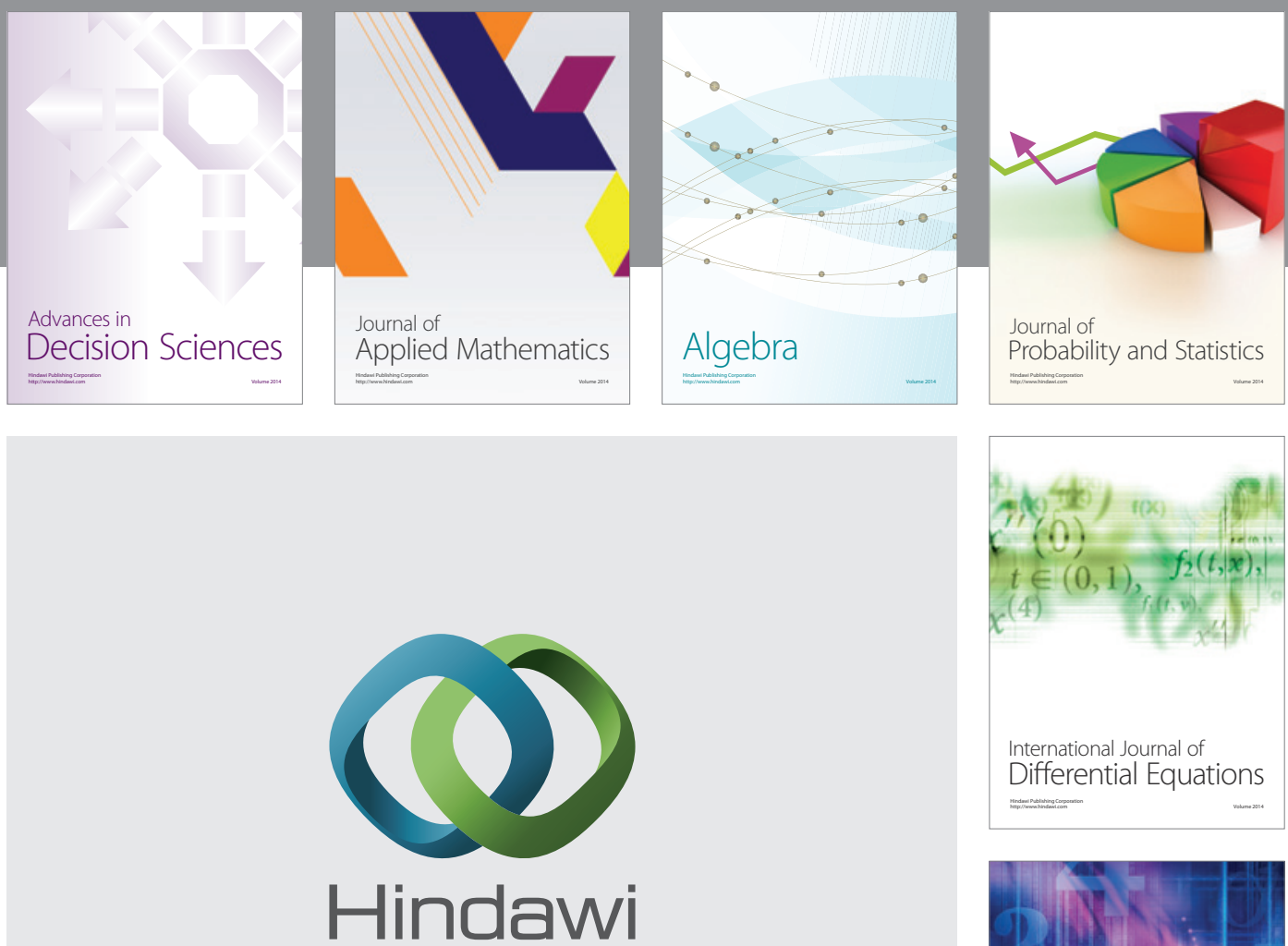

Submit your manuscripts at http://www.hindawi.com
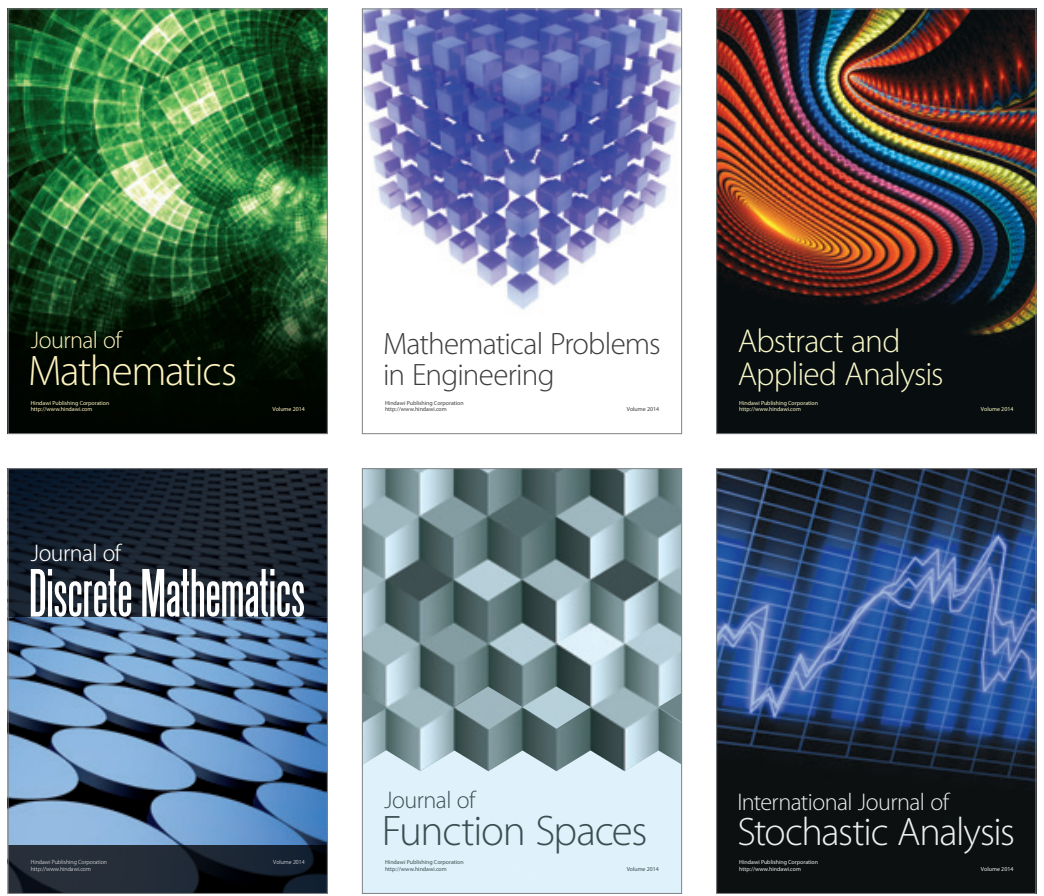

Journal of

Function Spaces

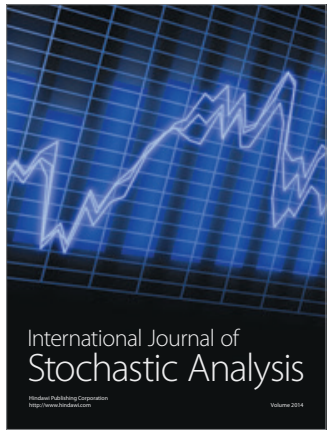

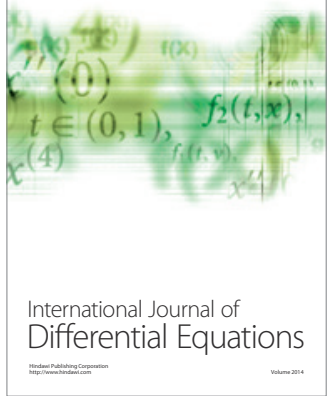
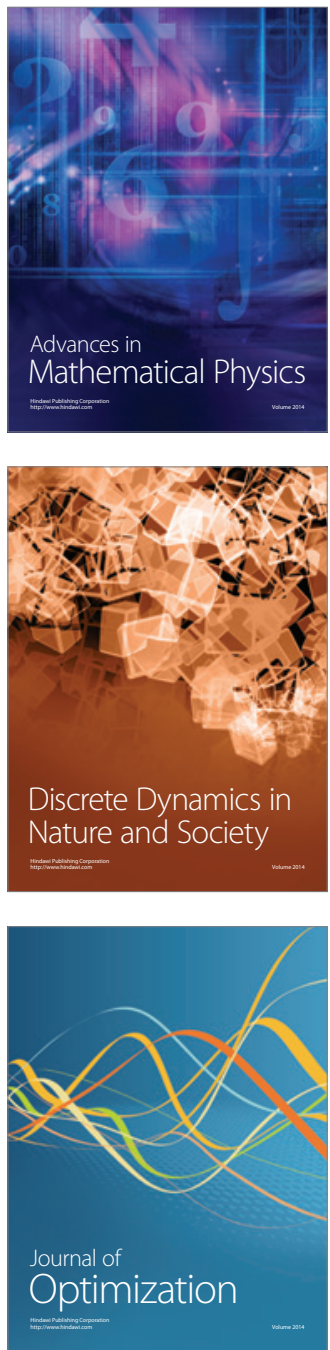